\title{
M. DUARTE
}

\section{Factores pronósticos en el linfoma B difuso de célula grande}

\section{Prognostic factors in diffuse large B-cell lymphoma}

Ver artículo: página 137

Dra. Mónica Duarte Romero: Especialista en Medicina Interna y Hematología. Profesor Titular Clínico, Facultad de Medicina, Universidad de los Andes. Docente Facultad de Medicina, Universidad El Bosque. Hematóloga Institucional Fundación Santa Fe de Bogotá. Bogotá, D.C. (Colombia).

E-mail:monicadarte2008@gmail.com
Dentro del grupo de linfomas no Hodgkin de tipo agresivo encontramos los linfomas B difusos de célula grande (LBDCG) bien reconocidos por sus características histopatológicas dentro de la clasificación de neoplasias linfoides de la Organización Mundial de la Salud (WHO 2008) (1-3) (Tabla 1). Los LBDCG corresponden del 30-40\% de los linfomas en adultos en la población occidental. Este grupo de linfomas comprende una variedad heterogénea de linfomas tanto por sus características clínicas como biológicas con un comportamiento clínico variable, que puede presentar respuesta y remisión a los tratamientos asociando rituximab en la mayoría de los casos y, sin embargo, por lo menos una tercera parte de los pacientes van a morir por esta enfermedad (4). En un gran esfuerzo por comprender mejor la fisiopatología y así mismo lograr una terapia más dirigida y con mejores resultados, se han desarrollado diferentes clasificaciones que permitan conocer, agrupar y predecir el comportamiento en la forma más homogénea posible de las diversas presentaciones de LBDCG.

La clasificación más reconocida a nivel mundial y validada en grandes series de pacientes con LBDCG, es la estratificación pronóstica basada en parámetros clínicos y bioquímicos que se encuentran fácilmente al alcance de toda evaluación clínica inicial, conocida como el Índice Pronóstico Internacional desarrollada por: "El proyecto internacional de factores pronósticos para linfoma no Hodgkin" publicado en 1993 que aún permanece vigente (5). Inicialmente se tuvieron en cuenta cinco factores pronósticos (Tabla 2): edad, estadío según la clasificación de Ann Arbor, nivel de deshidrogenasa láctica sérica, estado clínico general determinado por el "performance status" (Tabla 3 ) y el compromiso de sitios extranodales como parámetros para clasificar los pacientes en riesgo bajo, bajo intermedio, alto intermedio y alto. Posteriormente se propuso esta misma clasificación
Tabla 1. Clasificación de las enfermedades neoplásicas linfoides de la OMS 2008.

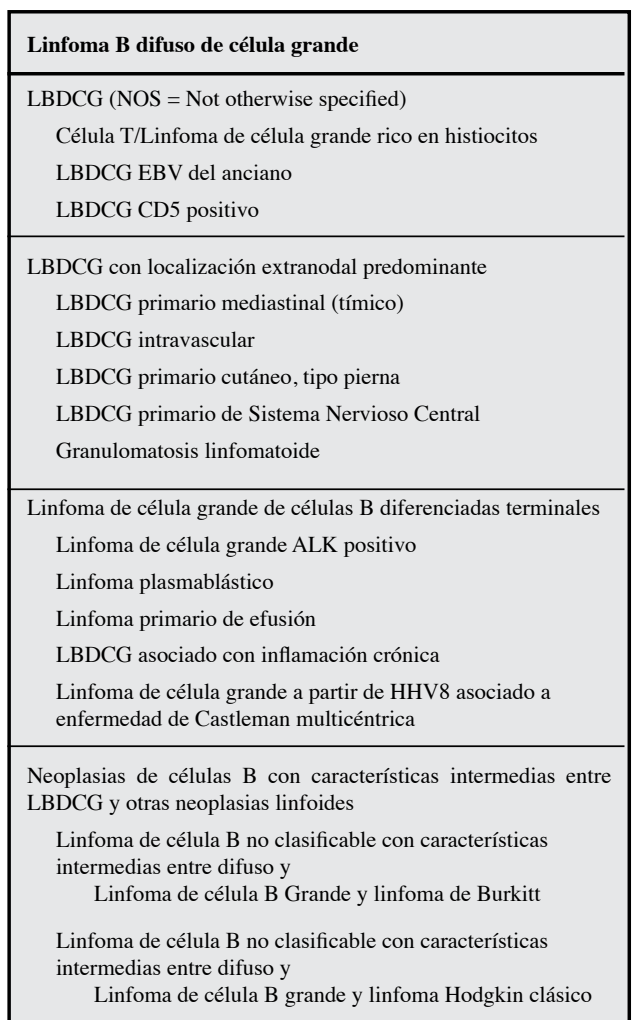

Tabla 2. Factores pronósticos (5).

\begin{tabular}{|l|c|}
\hline Parámetro & Factor de mal pronóstico \\
\hline Edad & $>60$ años \\
Estadío & III ó IV \\
Sitios extranodales & $\geq 2$ \\
Estado clínico & $\geq 2$ \\
(Performance status) & \\
Deshidrogenasa láctica & Aumentada \\
\hline
\end{tabular}

revisada conocida como revised IPI (6) en la que se evalúa la clasificación IPI en pacientes tratados con Rituximab Tabla 4. Teniendo en cuenta el comportamiento de las curvas de sobrevida, se reducen los grupos de riesgo a tres grupos pronósticos: muy buen pronóstico, bueno y mal pronóstico (6) Tabla 5. Mediante 
Tabla 3. Estado clínico (performance status)

\begin{tabular}{|c|l|}
\hline $\begin{array}{c}\text { Performance } \\
\text { estatus }\end{array}$ & \multicolumn{1}{c|}{ Definición } \\
\hline 0 & Asintomático \\
1 & Sintomático pero totalmente ambulatorio \\
2 & Sintomático y en cama $<50 \%$ del día \\
3 & Sintomático y en cama $>50 \%$ del día \\
4 & Postrado \\
\hline
\end{tabular}

Tabla 4. Factores pronósticos en el Indice Pronóstico Internacional IPI e IPI ajustado a la edad (5).

\begin{tabular}{|l|l|}
\hline IPI & IPI ajustado a la edad \\
\hline Edad $>60$ años & $\begin{array}{l}\text { Enfermedad avanzada: } \\
\text { III ó IV }\end{array}$ \\
Enfermedad avanzada: III ó IV & LDH aumentada \\
Compromiso extranodal & Performance status \\
$>1$ sitio & ECOG $\geq 2$ \\
LDH aumentada & \\
Performance status ECOG & \\
$\geq 2$ pobre & \\
\hline
\end{tabular}

estas clasificaciones se han logrado determinar pronósticos de sobrevida en grupos grandes de pacientes con LBDCG.

\section{Métodos moleculares}

Teniendo en cuenta los pacientes de mal pronóstico de la clasificación pronóstica IPI y ante la gran variabilidad del comportamiento clínico de los LBDCG con evolución rápidamente progresiva y en algunos casos desenlaces impredecibles, se requieren nuevos métodos diagnósticos que permitan abordar los diferentes mecanismos fisiopatológicos desde otras perspectivas biológicas para plantear alternativas terapéuticas apropiadas y dirigidas. Con el advenimiento de nuevas tecnologías, surgen otros caminos como la propuesta del proyecto de perfil molecular de leucemias y linfomas a comienzos de la década del 2000 en el que se caracterizan subtipos fenotípicos de LBDCG según el origen celular manifestado en el perfil de expresión génica (7). Se identifican los linfomas de tipo centrogerminal B (GCB) que expresan genes "normales" de las células B centrogerminales que corresponden a 50\% de los LBDCG, los linfomas B de fenotipo activado (ABC) que expresan genes que se observan en la activación de células en sangre periférica, corresponden a $30 \%$ de los LBDCG y un tercer grupo aproximadamente
$20 \%$ de los LBDCG denominado no clasificable conocido como "no GCB". Se reconoce al grupo $\mathrm{ABC}$ como el grupo de peor pronóstico. El pronóstico favorable de los linfomas GCB está asociado con la actividad disminuída de la vía de señal del factor nuclear $\mathrm{kB}$ que protege contra el efecto apoptótico de la quimioterapia; mientras que en el fenotipo $\mathrm{ABC}$ en el que se observa una activación constitutiva de esta vía que puede bloquear la apoptosis inducida por la quimioterapia, contribuyendo con el pobre pronóstico de este tipo de LBDCG $(7,8)$.

El fenotipo $\mathrm{ABC}$ se asocia con la activación de la vía de señal NF-kB, que se lleva a cabo mediante la dimerización de los factores de transcripción NF-kB en el citoplasma que migran a activar la transcripción de los genes blanco en el núcleo. Mediante métodos que reprimen la vía de señal $\mathrm{NF}-\mathrm{kB}$, se ha demostrado que los LBDCG dependen de esta vía de señal para sobrevivir, a diferencia de los LBDCG GCB. En los LBDCG ABC se encuentran alteraciones en los genes que codifican para el complejo de señal CBM, que incluyen CARD11, BCL10, MALT1 y que también hacen parte de la vía de señal del receptor de la célula $\mathrm{B}$ : $\mathrm{BCR}$; favoreciendo estimulación de la vía de señal NF-kB y alteraciones de la señal para el BCR. Por otra parte, se han detectado con mayor frecuencia mutaciones específicas de la subunidad CD79B que promueven activación crónica de señal para el BCR. Así mismo se han descrito otras alteraciones como amplificación 19q, deleción del locus tumor supresor INKa/ARF, trisomía 3, inactivación del tumor supresor BLIMP1 y la sobreexpresión de la familia de kinasas serina/treonina PIM, en relación con los LBDCG fenotipo ABC (9).

Tabla 5 (6). Índice pronóstico Internacional para Linfomas Agresivos.

\begin{tabular}{|l|c|c|c|}
\hline IPI estándar & Score IPI & $\begin{array}{c}\text { \% progresión libre } \\
\text { de enfermedad a 4 años }\end{array}$ & $\begin{array}{c}\text { \% sobrevida global } \\
\text { a 4 años }\end{array}$ \\
\hline Grupo de riesgo & 0 ó & 85 & 82 \\
\hline Bajo & 2 & 80 & 81 \\
Bajo intermedio & 3 & 57 & 49 \\
Alto intermedio & 4,5 & 41 & 59 \\
Alto & Score IPI & \% progresión libre & \% sobrevida global \\
\hline Índice pronóstico internacional revisado: & R-IPI años \\
\hline Pronóstico & 0 & 94 & 94 \\
\hline Muy bueno & 1,2 & 50 & 79 \\
Bueno & $3,4,5$ & 53 & 55 \\
Malo & \multicolumn{2}{|c|}{ de enfermedad a 4 años } & 94 \\
\hline
\end{tabular}


Para el fenotipo GCB se reconocen dos alteraciones genéticas: la translocación en BCL-2 t(14;18) y la amplificación C-REL que se presentan con una frecuencia de $25 \%$ y $15 \%$ respectivamente. La translocación $\mathrm{t}(14 ; 18)$ genera la expresión de la proteína antiapoptótica BCL2 que se encuentra en el centrogerminal $\mathrm{y}$ en pacientes tratados con rituximab se asocia con mal pronóstico en el fenotipo GCB a diferencia del ABC (9).

\section{Métodos inmunohistoquímicos}

Los LBDCG expresan marcadores pan B como CD19, CD20, CD22, CD79a y PAX5. La inmunoglobulina de superficie /citoplasmática ( $\operatorname{IgM}>\operatorname{IgG}>\operatorname{Ig} \mathrm{A})$ se encuentra presente de $50-75 \%$ de los casos. Otros marcadores incluyen: CD10 (endopeptidasa neutral asociada a la membrana que se expresa en las células centrogerminales y en tejido linfoide reactivo) en $40 \%$ de los casos, BCL-6 (proteína dedo de zinc que actúa como represora transcripcional y se expresa en células centrogerminales B y algunas células T CD4+) en 60\%, BCL2 en $50 \%$, CD43 en 20\%, IRF4/MUM1 (factor específico linfoide de la familia de los factores reguladores de interferón de los factores de transcripción, que se expresa en células plasmáticas y algunas células centrogerminales) hasta en 77\% y p53 (el gen supresor tumoral p53 actúa como factor multifuncional de transcripción involucrado en la detención del ciclo celular, apoptosis, diferenciación celular, reparación del DNA y mantenimiento de la estabilidad genómica) en $30 \%$ de los casos. La identificación de la proteína $M Y C$ nuclear se puede realizar mediante anticuerpo monoclonal, que se correlaciona positivamente con las translocaciones $M Y C$. Tanto la expresión de $M Y C$ como de BCL 2 son predictores independientes de menor sobrevida global y sobrevida libre de progresión (10).

Teniendo en cuenta las grandes exigencias en equipos, tecnología y costos de las diferentes pruebas moleculares, se desarrolló una forma más simplificada de identificar los factores pronósticos mediante métodos inmunohistoquímicos, utilizando algoritmos como el propuesto por Hans, que estratifica los linfomas en GCB o "no GC" según la expresión de los marcadores CD10, BCL6 y IRF4/MUM-1 (7, 8, 11). Los LBDCG que son $\mathrm{CD} 10+$ en más de $30 \%$ de las células, corresponden al grupo GCB; los casos que son CD10- y BCL6+, IRF4/MUM1-, también corresponden al grupo GCB. Los casos que son BCL6- CD10- ó BCL6+ y IRF4/MUM1+ son "no GCB". Este algoritmo pronóstico se correlaciona con el subtipo de perfil de expresión génica en algunos estudios hasta en un $86 \%$ de los casos (10).

Posteriormente se desarrollaron algoritmos con algunas modificaciones como el algoritmo de CHOI, en el que se incluye la expresión de FOXP1 [Forkhead boxprotein 1: es un factor de transcripción alas hélice que actúa como un represor transcripcional que se expresa tanto en tejido normal como tejido neoplásico y se expresa en células linfoides B pero no en células plasmáticas $(10,12)]$ y GCET1 [Germinal center B-cell expressed transcript-1 gene codifica para una proteína expresada en las células B centrogerminales (12)], que se correlacionado con el perfil de expresión génica en algunos estudios hasta en un $87 \%$ de los casos. También se describe el método de Tally que sustituye BCL-6 por LMO2 [LIM domain only 2: es un marcador de linfocitos centrogerminal, cuya función se desconoce (12)]; sin embargo, este método fue el menos sensible de todos $(9,10)$. El grupo de estudio internacional de LBDCG tratados con Rituximab-CHOP reportó un algoritmo basado en la expresión de CD10, FOXP1 y BCL6 que se correlacionó hasta en un 92,6\% de los casos con el perfil de expresión génica (13).

Las moléculas de reconocimiento inmunológico como la beta2microglobulina (componente de las moléculas de histocompatibilidad clase I presentes en las células nucleadas) y CD58 (molécula de adhesión expresada en las células presentadoras de antígeno especialmente en los macrófagos) se han identificado tanto en LBDCG de fenotipo GCB como de fenotipo ABC, lo que involucra el control inmunológico como otro mecanismo linfomagénico (9).

\section{El microambiente tumoral}

El microambiente tumoral se ha reconocido recientemente como uno de los factores pronósticos en el LBDCG más importantes. Se describen cuatro grupos pronósticos que involucran parámetros de expresión génica como el origen celular GCB, nódulo linfoide, el complejo mayor de histocompatibilidad clase II y la proliferación celular $(8,14)$. Como se menciona previamente el origen celular GCB se correlaciona con mejor pronóstico.

Las características del nódulo linfoide refle- 
jan el microamiente tumoral que se ha dividido en dos grupos: estroma-1 y estroma-2. Los genes que definen la firma estromal-1 codifican para componentes de la matriz extracelular como la fibronectina, osteonectina, isoformas de colágeno y laminina y factor antiangiogénico tromboespondina. También modificadores de la síntesis de colágeno como LOXL1 y SERPINH1, proteínas que remodelan la matriz extracelular como MMP2, MMP9, MMP14, PLAU y TIMP2 y factor de crecimiento de tejido conectivo (CTGF). Además incluye genes que se expresan en células de linaje monocítico como CEBPA y CSF2RA (15).

Los genes que definen la firma estromal-2 codifican para marcadores de células endoteliales como el factor de von Willebrand y CD31 (molécula de adhesión plaquetaria o PECAM1); genes que se expresan en el endotelio como EGFL7, MMRN2, GPR116, SPARCL1; genes reguladores de la angiogénesis como el receptor 2 del factor de crecimiento vascular endotelial (VEGF), GRB10 o factor receptor de crecimiento que se une a la proteína 10 y media la vía de señal KDR; integrina alfa 9 que desencadena la señal para VEGF; la tirosina kinasa endotelial (TEK), el receptor de la tirosina kinasa para la citoquina angiopoyetina; ROBO4 molécula endotelial específica que regula angiogénesis y el oncogen homólogo del virus de la eritroblastosis E26 (ERG) factor de transcripción necesario en la formación túbulo endotelial. Otros genes como CAV1, CAV2 y EHD2 codifican componentes de caveolae que se especializan en las estructuras de membrana plasmática abundantes en las células endoteliales necesarias para la angiogénesis (15).

En caso de alta representación de componente estromal tipo 1 , se correlaciona con gran depósito de matriz extracelular e infiltración por monocitos y macrófagos que predice buen pronóstico; mientras que el componente estromal tipo 2 refleja angiogénesis y gran densidad vascular en el estroma tumoral que se relaciona con mal pronóstico $(14,15)$. Con el fin de evaluar el tipo estromal 1 se utiliza un anticuerpo dirigido contra la proteína secretada, ácida y rica en histidina SPARC: si se encuentra una tinción SPARC muy positiva en el estroma tumoral, se correlaciona con un mejor pronóstico comparado con los linfomas SPARC cuya tinción es escasa o ausente (16). Otro método reportado consiste en medir la densidad de los microvasos, lo que se relaciona con mal pronóstico aún en pacientes tratados con esquemas de quimioterapia asociando rituximab (17).

La pérdida del complejo mayor de histocompatibilidad clase II (HLA-DRA) fue identificada como un factor pronóstico independiente que se correlaciona con la pérdida de la respuesta celular inmunológica dada por los linfocitos CD8+ y por ende con mal pronóstico. En la respuesta inmunológica los linfocitos citotóxicos CD8+ reaccionan frente a antígenos presentados por moléculas del complejo mayor de histocompatibilidad clase I que se encuentran en casi todas las células nucleadas. Los antígenos del complejo mayor de histocompatibilidad clase II sólo se presentan por algunas células presentadoras de antígenos como células $\mathrm{B}$, macrófagos y células dendríticas generando una respuesta celular T ayudadora CD4+. La respuesta celular $\mathrm{T}$ ayudadora contribuye con la respuesta celular T citotóxica que es la respuesta celular inmunológica asociada con la vía final encargada de la muerte celular tumoral (18).

La proliferación celular se manifiesta clínicamente por el estado avanzado del compromiso clínico y biológicamente por marcadores como el porcentaje del Ki67. El Ki67 es un antígeno nuclear que se expresa en las células en ciclo celular y el porcentaje de las células que expresan Ki 67 se correlaciona con el volumen de células tumorales. No se considera marcador pronóstico independiente en pacientes tratados con rituximab $(14,15)$.

\section{MicroRNAs}

Los MicroRNAs además de tener un rol en el diagnóstico de los LBDCG también se consideran con potencial pronóstico. Aún no son muchos los microRNA identificados con los fenotipos de LBDCG; sin embargo, miR-21 se ha establecido como un diferenciador entre el fenotipo GCB y ABC. Niveles aumentados de miR-21 se asocian con mayor sobrevida libre de enfermedad. En pacientes con LBDCG de fenotipo ABC y alta expresión de MiR-155 se encontró una mayor sobrevida (19).

Otros microRNA identificados como factores pronósticos independientes en el seguimiento de pacientes con LBDCG tanto para la sobrevida global a cinco años como la sobrevida libre de enfermedad fueron: miR-18a, miR-181a y miR- 222 (19). En la actualidad los microRNA clasificados por grupos se encuentran en evaluación tanto en 
la patogénesis de los linfomas como en su valor predictivo.

\section{Doble hit y triple hit}

La proliferación celular asociada a $M Y C$ se correlaciona con mal pronóstico $(14,20,21)$. Las translocaciones que comprometen $M Y C$ se observan hasta en $10 \%$ de los casos (20). MYC se localiza en el cromosoma $8 \mathrm{q} 24$ y codifica para un factor de transcripción involucrado en la regulación de la procesos celulares como el control del ciclo celular, la proliferación, metabolismo, apoptosis y migración celular. Se define como un factor pronóstico adverso independiente en los pacientes con LBDCG tratados con rituximab (14). La presencia de MYC no pudo ser correlacionada con los factores pronósticos clínicos ni con la proliferación celular medida por Ki67 (20).

Los linfomas B que presentan simultáneamente rearreglos en $M Y C$ y en IGH-BCL2 se conocen como linfomas doble hit y se caracterizan por su comportamiento clínico agresivo asociado a alteraciones cromosómicas complejas, alta proliferación celular con Ki67 superior a $80 \%$ y muy pobre pronóstico con una sobrevida media inferior a 1 año $(3,14$, $21,22)$. El inmunofenotipo de los LBDCG doble hit se presenta usualmente como fenotipo GCB con expresión de CD10+, BCL6+ y IRF4/MUM1-. La proteína BCL2 se detectó en 95\% de los casos (22). Los linfomas triple hit se presentan asociando compromiso de $M Y C$, BCL2 y BCL6 $(21,22)$.

\section{CD5}

CD5 es una glicoproteína transmembrana que se expresa en células $\mathrm{T}$ normales y en un grupo de células B neoplásicas (12). Los LBDCG CD5+ tienen un comportamiento clínico más agresivo, generalmente se trata de pacientes mujeres mayores con enfermedad retroperitoneal de tipo bulky. Se presentan de novo y no se relacionan con proliferación linfocitaria crónica CD5+ como leucemia linfoide crónica o con linfoma del manto. La agresividad del linfoma parece dependiente de la activación del CD5 (3). Sin embargo la mayoría de los LBDCG CD5+ pertenecen al fenotipo $\mathrm{ABC}$, generalmente $\mathrm{CD} 10-, \mathrm{Bcl} 2+$ y MUM1+. Se asocian con factores de mal pronóstico como LDH elevada, síntomas B, compromiso extranodal frecuente y en particular del SNC, estado clínico avanzado y pobre performance status. El tratamiento con rituxi- mab parece haber disminuído el compromiso del SNC. También se identifican alteraciones cromosómicas complejas (23).

\section{Conclusiones}

La aplicación del indice pronóstico internacional sigue siendo aún una clasificación muy valiosa a pesar de que fue desarrollada antes del tratamiento de rutina con rituximab para todos los pacientes con LBDCG. En la nueva era de tratamiento aún no se han validado las diferentes tentativas de estratificación pronóstica, aunque el aporte al conocimiento sobre el comportamiento biológico de los diferentes LBDCG ha sido considerable con estas nuevas tecnologías. Aún en la actualidad no se pueden aplicar estos métodos moleculares de rutina en la práctica clínica debido no solamente a que estos parámetros carecen de estandarización que permita su validez a gran escala, sino también por tratarse de recursos de alto costo que requieren laboratorios de alta tecnología. Teniendo en cuenta que los métodos inmunohistoquímicos constituyen una herramienta de menor costo, se vienen aplicando más ampliamente en la práctica clínica.

Todas estas posibilidades de identificación de factores pronósticos, permiten desarrollar estrategias terapéuticas dirigidas como tratamientos antiangiogénicos en el caso de linfomas con densidad vascular aumentada como por ejemplo lenalidomida o como bevacizumab que es un anticuerpo monoclonal dirigido contra el factor de crecimiento endotelial (VEGF) actualmente en investigación $(9,24)$; de estimulación inmunológica celular T en el caso de linfomas con disminución de la capacidad de respuesta inmunológica mediante activación de células $\mathrm{T}$ in vitro; entre otras.

Según la biología tumoral se vienen investigando anticuerpos monoclonales dirigidos contra otras molélulas de los LBDCG como el epratuzumab anticuerpo monoclonal humanizado dirigido contra el marcador célula $\mathrm{B}$ CD22; dacetuzumab anticuerpo monoclonal anti - CD40 siendo CD40 una proteína transmembrana de la superfamilia del receptor del factor de necrosis tumoral expresada en células $\mathrm{B}$; blinatumomab un bi-anticuerpo dirigido contra el marcador B CD19 y el marcador T CD3; inotuzumab ozogamicin dirigido contra CD22 conjugado con calicheamicina (24). También se utilizan moléculas inhibidoras de marcadores específicos como BCL6 (25). En el caso del LB$\mathrm{DCG}$ de fenotipo $\mathrm{ABC}$ se viene investigando la 
asociación de esquemas de quimioterapia con bortezomib inhibidor del proteasoma Barton y más recientemente el inhibidor de la tirosina kinasa de la agamaglobulinemia de Bruton (BTK) conocido como ibrutinib asociado a lenalidomida (26).

Los marcadores de mal pronóstico detectados en el estudio de Guevara y cols (27), están correlacionados con pacientes con enfermedad avanzada y con el fin de considerarlos como factores pronósticos aislados, deben ser corroborados con el desenlace clínico y los marcadores biológicos que actualmente se encuentran en investigación; teniendo en cuenta que en estudios clínicos previos, el ki67 y la beta2microglobulina no han sido validados como factores pronósticos aislados en pacientes tratados con rituximab y pueden explicarse únicamente por el diagnóstico tardío de la enfermedad $(12,14,15)$.

Estos esfuerzos permitirán mejores resultados con tratamientos más dirigidos y ajustados a pacientes con LBDCG según sus características clínicas, biológicas y moleculares; teniendo en cuenta que todo tratamiento incluyendo la adición de rituximab puede mejorar la respuesta tumoral pero además de aumentar costos también comporta toxicidad adicional asociada a desenlaces fatales que debe ser evaluada con modelos de seguimiento estricto (28).

\section{Referencias}

1. Swerdlow SH, Campo E, Harris NL, Jaffe ES, Pileri SA, Stein $\mathbf{H}$, et al. Who classification of tumors of haematopoietic and lymphoid tissues. 4th ed. Lyon: IARC Press; 2008.

2. Campo E, Swerdlow SH, Harris NL, Pileri S, Stein H, Jaffe ES. The 2008 WHO classification of lymphoid neoplasms and beyond: evolving concepts and practical applications. Blood $2011 ; 117$.

3. Jaffe ES, Pittaluga S. Aggressive B-cell lymphomas: a review of new and old entities in the WHO classification. Hematology Am Soc Hematol Educ Program 2011; 2011: 506-14.

4. Sehn LH. Paramount prognostic factors that guide therapeutic strategies in diffuse large B-cell lymphoma. Hematology Am Soc Hematol Educ Program 2012;2012:402-9.

5. A predictive model for agressive non-Hodgkin's lymphoma: The international non-Hodgkin's lymphoma prognostic factors project. N Engl J Med 1993; 329 (14). 987-94.

6. Sehn LH, Berry B, Chhanabhai M, Fitzgerald C, Gill K, Hoskins P, et al. The revised International Prognostic Index (R-IPI) is a better predictor outcome than the standard IPI for patients with diffuse large B cell-lymphoma treated wiht RCHOP. Blood 2007;109 (5):1857 - 61.

7. Alizadeh AA, Eisen MB, Davis RE, Ma C, Lossos IS, Rosenwald A, et al. Distinct types of diffuse large B-cell lymphoma identified by gene expression profiling. Nature 2000; 403 : 503-11.

8. Rosenwald A, Wright G, Chan WC, Connors JM, Campo E, Fisher RI, et al. Lymphoma/Leukemia Molecular Profiling Project. The use of molecular profiling to predict survival after chemotherapy for diffuse large B-cell lymphoma. N Engl J Med 2002 Jun 20; 346 (25): 1937 -47.

9. Barton S, Hawkes EA, Wotherspoon A, Cunningham D. Are we ready to stratify treatment for diffuse large B-cell lymphoma using molecular hallmarks? Oncologist 2012;17(12):1562-73.
10. Said JW. Aggressive B-cell lymphomas: how many categories do we need? Mod Pathol 2013 Jan;26 Suppl 1:S42-56.

11. Hans CP, Weisenberger DD, Greiner TC, Gascoyne RD, Delabie J, Ott G, Müller-Hermelink HK, Campo E, Braziel RM, Jaffe ES, Pan Z, Farinha P, Smith LM, Falini B, Banham AH, Rosenwald A, Staudt LM, Connors JM, Armitage JO, Chan WC. Confirmation of the molecular classification of diffuse large B-cell lymphoma by immunohistochemistry using a tissue microarray. Blood 2004; 103: 275 -282.

12. Ninan MJ, Wadhwa PD, Gupta P.Prognostication of diffuse large B-cell lymphoma in the rituximab era. Leuk Lymphoma 2011 Mar; 52(3): 360-73.

13. Visco C, Li Y, Xu-Monette ZY RN, Green TM, Li Y, Tzankov A, et al. Comprehensive gene expression profiling and immunohistochemical studies support application of immunophenotypic algorithm for molecular subtypeclassification in diffuse large B-cell lymphoma: a report form the International DLBCL Rituximab-CHOP Consortium Program Study. Leukemia 2012; 2103-2113.

14. Perry AM, Mitrovic Z, Chan WC. Biological prognostic markers in diffuse large B-cell lymphoma. Cancer Control $2012 \mathrm{Jul} ; 19$ (3):214-26.

15. Lenz G, Wright G, Dave SS, Xiao W, Powell J, Zhao H, et al.Lymphoma/Leukemia Molecular Profiling Project. Stromal gene signatures in large B-cell lymphomas. N Engl J Med 2008; 359 (22): 2313 -2323.

16. Meyer PN, Fu K, Greiner T, Smith L, Delabie J, Gascoyne R, et al. The stromal cell marker SPARC predicts for survival in patients with diffuse large B-cell lymphoma treated with Rituximab. Am J Clin Pathol 2011; 135 (1):54-61.

17. Cardessa-Zalzmann TM, Colomo L, Gutiérrez G, Chan WC, Weisenberger D, Climent F, et al. High microvessel density determines a poor outcome in patients with diffuse large B-cell lymphoma treated with Rituximab plus chemotherapy. Haematologica 2011; 96(7): 996-1001.

18. Rimsza LM, Roberts RA, Miller TP, Unger JM, LeBlanc M, Braziel RM, et al. Loss of MHC class II gene and protein expression in diffuse large B-cell lympho- ma is related to decreased tumor immu- nosurveillance and poor patient survival regardless of other prognostic factors: a follow-up study from the Leukemia and Lymphoma Molecular Profiling Project. Blood 2004;103:4251-8.

19. Troppan K, Wenzl K, Deutsch A, Ling H, Neumeister $P$, Pichler M. MicroRNAs in diffuse large B-cell lymphoma: implications for pathogenesis, diagnosis, prognosis and therapy. Anticancer Res 2014 Feb; 34(2): 557-64.

20. Savage KJ, Johnson NA, Ben-Neriah S, Connors JM, Sehn LH, Farinha P, et al. MYC gene rearrangements are associated with a poor prognosis in diffuse large B-cell lymphoma patients treated with R-CHOP chemotherapy. Blood 2009 Oct 22; 114(17): 3533-7.

21. Lin P, Medeiros LJ. The impact of MYC rearrangements and "double hit" abnormalities in diffuse large B-cell lymphoma. Curr Hematol Malig Rep 2013 Sep; 8(3): 243-52.

22. Aukema SM, Siebert R, Schuuring E, van Imhoff GW, Kluin-Nelemans HC, Boerma EJ, et al. Double-hit B-cell lymphomas. Blood $2011 \mathrm{Feb} 24$; 117(8):2319-31.

23. Jain P, Fayad LE, Rosenwald A, Young KH, O>Brien S. Recent advances in de novo CD5+ diffuse large B cell lymphoma. Am J Hematol 2013 Sep;88(9):798-802.

24. Cultrera JL, Dalia SM. Diffuse large B-cell lymphoma: current strategies and future directions. Cancer Control 2012 Jul; 19(3): 204-13.

25. Pasqualucci L. The genetic basis of diffuse large B-cell lymphoma. Curr Opin Hematol 2013 Jul; 20(4): 336-44.

26. Staudt LM. II. Therapy of DLBCL based on genomics. Hematol Oncol 2013 Jun; 31 (Suppl 1):26-8.

27. Guevara NM, Jaramillo PE, Rendón J, Gaviria LM. Caracterización de factores pronósticos al diagnóstico de pacientes con Linfoma B difuso de células grandes en un Hospital Universitario, 2009-2012. Acta Med Colomb 2014; 39: 137-147.

28. Lindenmeyer LP, Hegele V, Caregnato JP, Wüst D, Grazziotin L, Stoll P. Follow-up of patients receiving rituximab for diffuse large B cell lymphoma: an overview of systematic reviews. Ann Hematol 2013 Nov; 92(11): 1451-9. 